\title{
Imaging of Endolymphatic and Perilymphatic Fluid at 3T After Intratympanic Administration of Gadolinium-Diethylene-Triamine Pentaacetic Acid
}

\author{
S. Naganawa \\ M. Sugiura \\ M. Kawamura \\ H. Fukatsu \\ M. Sone \\ T. Nakashima
}

SUMMARY: By optimizing the inversion time of a 3D inversion-recovery turbo spin-echo sequence at 3T, we obtained separate images of endolymphatic and perilymphatic space 24 hours after intratympanic administration of gadolinium contrast material. In patients with Ménière disease, endolymphatic hydrops were detected not only in the cochlea but also in the vestibule. Fusion of the 2 types of images visualized the entire fluid space of the labyrinth and the spatial relationship of the 2 spaces.
A fter intratympanic injection of gadolinium-diethylenetriamine pentaacetic acid (Gd-DTPA) in an animal study, Gd-DTPA was absorbed through the round window membrane and was distributed mainly into the perilymphatic space of the labyrinth. ${ }^{1}$ Enlarged endolymphatic space in patients with Ménière disease has been successfully recognized as an area with low signal intensity partly surrounded by high-signal intensity perilymphatic fluid on 3D-fluid-attenuated inversion recovery (FLAIR) images obtained after intratympanic injection of Gd-DTPA. ${ }^{2}$ However, the boundary between the cochlear endolymphatic space and surrounding bone was not clear, as both had low signal intensity on 3D-FLAIR images. To visualize endolymphatic space in the labyrinth as high signal intensity, while maintaining the differentiation from perilymphatic fluid space, we selected an inversion time shorter than that of 3D-FLAIR to suppress the signal intensity of perilymphatic fluid with a higher concentration of Gd-DTPA.

\section{Materials and Methods}

\section{Patients}

A total of 4 patients ( 3 with clinically diagnosed Ménière disease and 1 with sudden sensorineural hearing loss, ages 38-69 years; 2 men and 2 women) underwent intratympanic administration of GdDTPA bis-methylamide (Gd-DTPA-BMA, Omniscan; Daiichi Pharmaceutical, Tokyo, Japan). These patients were scheduled for intratympanic injection therapy with gentamicin (for the 3 patients with Ménière disease) or with a steroid (for the patient with sudden sensorineural hearing loss). We obtained written informed consent from all patients. The institutional review board of our university hospital approved our study.

\section{Intratympanic Gadolinium Injection}

The detailed methods for intratympanic gadolinium injection have been reported previously. ${ }^{2}$ According to the results from this previous study, scan delay after intratympanic gadolinium injection was determined as 24 hours to allow the distribution of gadolinium widely in the perilymphatic space of the labyrinth.

\section{Received June 15, 2007; accepted after revision October 2.}

From the Departments of Radiology (S.N., M.K., H.F.) and Otorhinolaryngology (M.S., M.S., T.N.), Nagoya University Graduate School of Medicine, Nagoya, Japan.

Please address correspondence to Shinji Naganawa, MD, Department of Radiology, Nagoya University Graduate School of Medicine, 65 Tsurumai-cho, Shouwa-ku, Nagoya 466-8550, Japan; e-mail: naganawa@med.nagoya-u.ac.jp

DOl 10.3174/ajnr.A0894
Gd-DTPA-BMA was diluted eightfold with saline (v/v 1:7). We injected the diluted Gd-DTPA-BMA intratympanically through the tympanic membrane using a 23 -gauge needle and a 1-mL syringe after the patient was placed in the supine position with their head turned approximately $30^{\circ}$ away from the sagittal line toward the healthy ear. The diluted Gd-DTPA-BMA was injected until a backflow of fluid into the external ear was observed through a microscope, resulting in an injected volume of 0.4 to $0.5 \mathrm{~mL}$ per patient. After the injection, the patients remained in the supine position for 60 minutes with the head turned approximately $60^{\circ}$ away from the sagittal line toward the healthy ear.

\section{MR Imaging}

We performed all scans on a 3T MR imaging scanner (Magnetom Trio; Siemens, Erlangen, Germany) using a receive-only 12-channel phased-array coil. T1-weighted 3D-fast low-angle shot (FLASH) and conventional 3D-FLAIR imaging were acquired 24 hours after intratympanic injection of diluted Gd-DTPA-BMA. In addition, T2weighted 3D-constructive interference in the steady state (CISS) imaging was performed to obtain reference images of labyrinthine fluidspace anatomy.

The parameters for 3D-FLASH were as follows: TR, $4.3 \mathrm{~ms}$; TE, $1.97 \mathrm{~ms}$; flip angle, $10^{\circ}$ with radio frequency spoiling; matrix size, $256 \times 256$; and 96 axial 0.8 -mm-thick sections covering the posterior fossa with a $16-\mathrm{cm}$ square FOV. The NEX was 2, giving a total scan time of 2 minutes 51 seconds.

The parameters for 3D-CISS were as follows: TR, $11.42 \mathrm{~ms}$, TE, $5.71 \mathrm{~ms}$; flip angle, $50^{\circ}$; matrix size, $320 \times 320$; and 48 axial $0.8-\mathrm{mm}-$ thick sections with a $16-\mathrm{cm}$ square FOV. The NEX was 1 , and the scan time was 3 minutes 42 seconds.

The parameters for 3D-FLAIR were as follows: TR, $9000 \mathrm{~ms}$; TE, $128 \mathrm{~ms}$; flip angle, $180^{\circ}$ (constant) for the turbo spin-echo refocusing echo-train; echo-train length, 23; matrix size, $384 \times 384$; and 12 axial 2 -mm-thick sections covering the labyrinth with a $16-\mathrm{cm}$ square FOV acquired with use of the generalized autocalibrating partially parallel acquisition parallel imaging technique with an acceleration factor of 2. ${ }^{3}$ The NEX was 1 , and the scan time was 14 minutes.

In the first 2 patients, $2 \mathrm{D}$ inversion-recovery (IR) turbo spin-echo imaging with TR, TE, and echo-train length identical to those of the 3D-FLAIR protocol was performed with various inversion times (2300, 2100, 1900, 1700, 1500, 1300, 1100, 900, 700, and $500 \mathrm{~ms})$ to determine the null point of perilymphatic fluid containing a low concentration of Gd-DTPA-BMA at 24 hours after intratympanic injection. In both patients, 2D-IR with inversion times of 900 and $1100 \mathrm{~ms}$ 

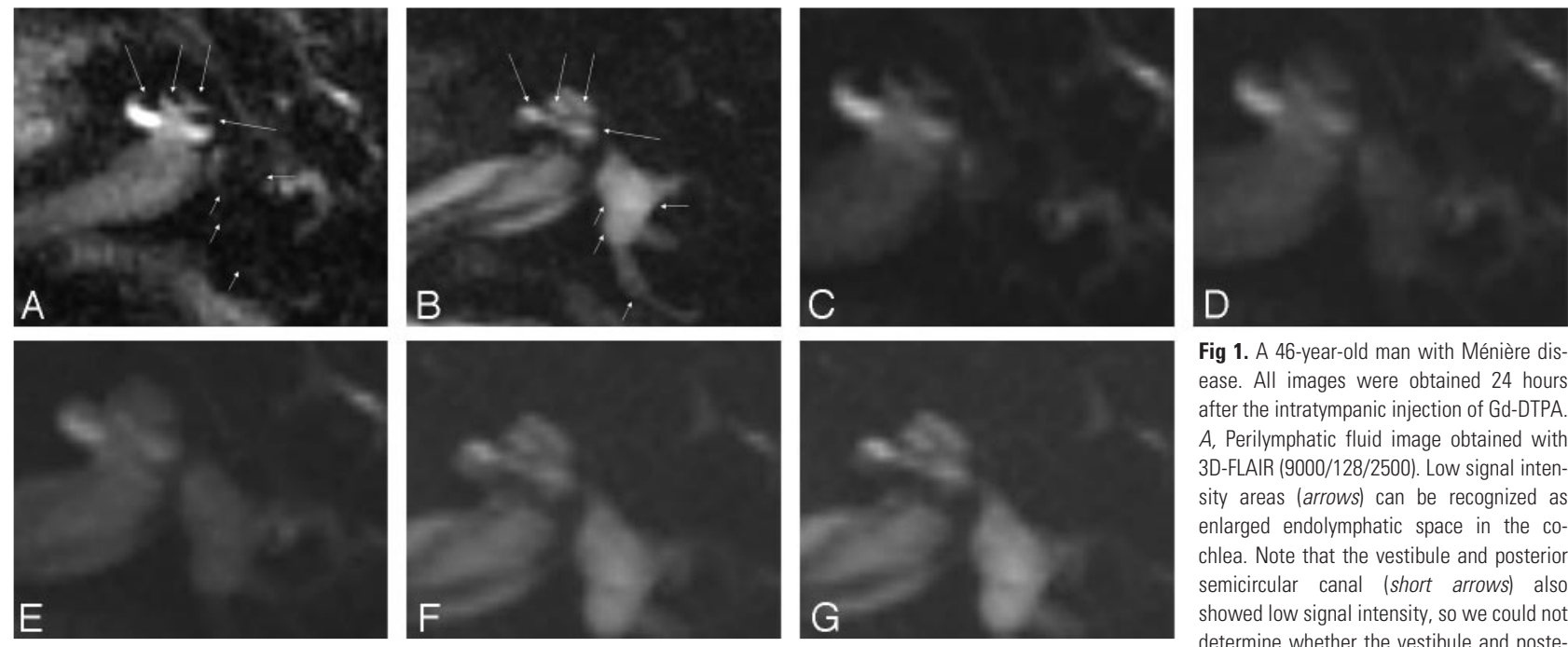

Fig 1. A 46-year-old man with Ménière disease. All images were obtained 24 hours after the intratympanic injection of Gd-DTPA $A$, Perilymphatic fluid image obtained with 3D-FLAIR (9000/128/2500). Low signal intensity areas (arrows) can be recognized as enlarged endolymphatic space in the cochlea. Note that the vestibule and posterior semicircular canal (short arrows) also showed low signal intensity, so we could not determine whether the vestibule and posterior semicircular canal were filled with endolymphatic fluid. $B$, Endolymphatic fluid image obtained with a 3D inversion-recovery sequence (9000/128/1000). Endolymphatic space in the cochlea (arrows) shows high signal intensity on this image. This image confirmed that the vestibule and posterior semicircular canal were filled with fluid. $C-G$, Fusion of a perilymphatic fluid image $(C)$ and an endolymphatic fluid image $(G)$ with transitional images $(D-F)$. By changing the fusion mixture rate on a workstation, the spatial relationship between perilymphatic and endolymphatic space was easily appreciated in both the cochlea and vestibule. In this case, endolymphatic space was enlarged in both the cochlea and vestibule, but the enlargement was especially prominent in the vestibule. Note that CSF in the internal auditory canal is visualized as high signal intensity on the endolymphatic fluid image (G). The signal intensity of perilymphatic space is just suppressed. Thus, the term endolymphatic image is only useful for labyrinthine space.
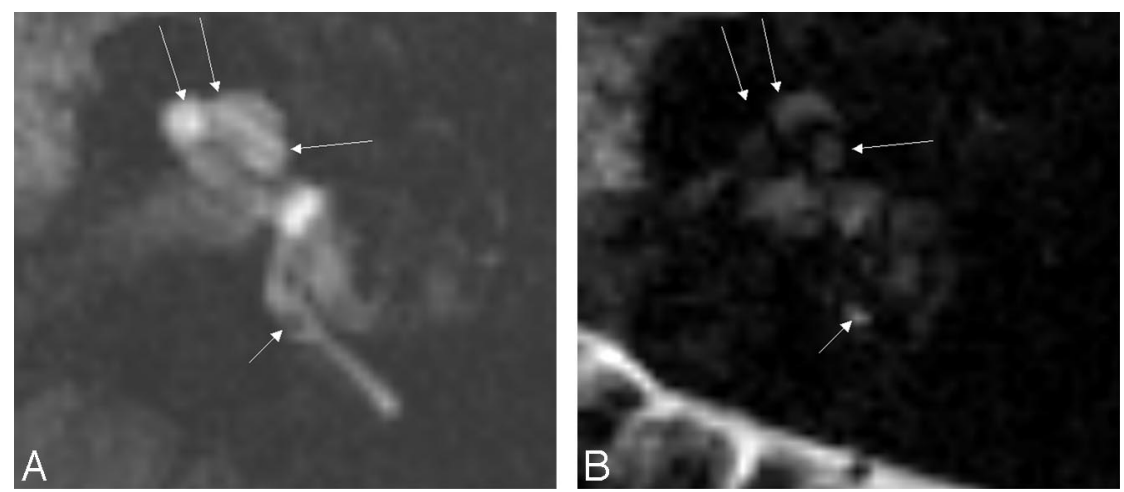

Fig 2. A 43-year-old woman with sudden sensorineural hearing loss in the left ear. All images were obtained 24 hours after the intratympanic injection of Gd-DTPA. A, Perilymphatic fluid image obtained with 3D-FLAIR (9000/128/2500). Areas of low signal intensity in the position of the cochlear duct (arrows) cannot be recognized as the endolymphatic space in the cochlea, probably because of their small size. Note that the endolymphatic space of the posterior ampulla (short arrow) showed low signal intensity. B, Endolymphatic fluid image obtained with a $3 D$ inversion-recovery sequence $(9000 / 128 / 1000)$. The endolymphatic space in the cochlea (arrows) cannot be recognized as high signal intensity on this image. Note that the endolymphatic space of the posterior ampulla (short arrow) showed high signal intensity alternatively compared with Fig $2 A$.

showed the lowest signal intensity in the perilymphatic space. Thus, in all 4 subjects, an inversion time of $1000 \mathrm{~ms}$ was selected for 3D-IR imaging of endolymphatic space. Other 3D-IR parameters for endolymphatic imaging were identical to those of 3D-FLAIR. If the size of the endolymphatic space was enlarged, endolymphatic and perilymphatic images were reviewed while referring to 3D-CISS images. The cochlear endolymphatic space is thought to be enlarged if the cochlear duct (endolymphatic space in the cochlea) is bulging toward the perilymphatic space in the scala vestibuli. Also, the vestibular endolymphatic space is thought to be enlarged if the endolymphatic space is larger than the perilymphatic space.

\section{Image Fusion}

Endolymphatic (3D-IR with an inversion time of $1000 \mathrm{~ms}$ ) and perilymphatic images (3D-FLAIR) were fused on a Leonardo workstation (Siemens, Erlangen, Germany). To confirm that the spatial relationship between the perilymphatic and endolymphatic images was anatomically correct, we viewed the fused images on the workstation monitor while changing the weighting scale continuously from pure 3D-IR to pure 3D-FLAIR.

\section{Results}

In all patients, an area of low signal intensity in the labyrinth on 3D-FLAIR showed high signal intensity on endolymphatic images (Fig $1 A, B$ ). By scaling the weighting on a fused image, the spatial relationship between the endolymphatic and perilymphatic images was clearly discernible (Fig $1 C-G$ ). In the 3 patients with Ménière disease, the endolymphatic space seemed to be enlarged. In the patient with sudden hearing loss, no enlargement of the endolymphatic space was noted (Fig 2).

\section{Discussion}

Many researchers ${ }^{4}$ have attempted separate visualization of perilymphatic and endolymphatic fluid space with MR imaging. Direct visualization of the Reissner membrane with use of high spatial-resolution imaging was successful in animals ${ }^{5}$ and human cadavers ${ }^{6,7}$; however, clear visualization in a living human subject has not been successful. Four hours after intravenous administration of Gd-DTPA in healthy human volunteers, a slight increase in signal intensity was noted in the labyrinth. ${ }^{8}$ However, probably because of a gadolinium concentration that was too low in the perilymphatic space, differentiation between the endolymphatic and perilymphatic space was not achieved. Intratympanic injection of Gd-DTPA and the application of 3D-FLAIR at 3T made the visualization of endolymphatic hydrops possible in vivo. ${ }^{2}$ Although intratympanically administered Gd-DTPA distributes mainly into the perilymphatic fluid space and not into the endolymphatic 
space, on 3D-FLAIR the hypointense endolymphatic space was difficult to differentiate from the surrounding bone and air. To delineate the endolymphatic space more clearly and to allow the quantification of the endolymphatic-space volume in the future, the endolymphatic space needs to be visually differentiated not only from the perilymphatic space but also from bone and air. By changing the inversion time, it was possible to separately visualize the endolymphatic and perilymphatic spaces as positive signal intensity. This will allow volume quantification of each space in the future if the spatial resolution is improved. Quantification of each space is important for the objective diagnosis of endolymphatic hydrops and for the monitoring of treatment effectiveness.

Fusion techniques convinced us that each space was separately visualized not only in the cochlea, but also in the vestibule and semicircular canals.

In our study, the labyrinth without intratympanic gadolinium injection (the other side ear) showed uniformly high signal intensity of the whole labyrinth on endolymphatic imaging with use of $3 \mathrm{D}-\mathrm{IR}$. This means that the suppression of perilymph on endolymphatic imaging by the present method in the injected side is based on the distribution of gadolinium.

One of the limitations of our presented method was the long acquisition time, 30 minutes for 2 kinds of lymphatic space images. One possibility to obtain endolymphatic images in a shorter period of time is the subtraction of perilymphaticspace images from T2-weighted images. This might allow us to obtain endolymphatic-space images if the subject is nearly motionless during the scans.
The other limitation of our method was the potential rupture of Reissner membrane in a very advanced case of Ménière disease. In such case, contamination of the endolymph and perilymph would occur and endolymphatic space might show low signal intensity on endolymphatic imaging.

\section{Conclusion}

By optimizing the inversion time, it was possible to obtain images of the endolymphatic and perilymphatic spaces. The endolymphatic space was differentiated not only from the perilymphatic space, but also from bone and air. This method might open the door to objective evaluation of endolymphatic-space disease.

\section{References}

1. Zou J, Pyykk[ddot]o I, Bjelke B, et al. Communication between the perilymphatic scalae and spiral ligament visualized by in vivo MRI. Audiol Neurootol 2005; 10:145-52

2. Nakashima T, Naganawa S, Sugiura M, et al. Visualization of endolymphatic hydrops in patients with Meniere's disease. Laryngoscope 2007;117:415-20

3. Griswold MA, Jakob PM, Heidemann RM, et al. Generalized autocalibrating partially parallel acquisitions (GRAPPA). Magn Reson Med 2002;47:1202-10

4. Niyazov DM, Andrews JC, Strelioff D, et al. Diagnosis of endolymphatic hydrops in vivo with magnetic resonance imaging. Otol Neurotol 2001;22:813-17

5. Koizuka I, Seo Y, Murakami M, et al. Micro-magnetic resonance imaging of the inner ear in the guinea pig. NMR Biomed 1997;10:31-34

6. Koizuka I, Seo R, Kubo T, et al. High-resolution MRI of the human cochlea. Acta Otolaryngol Suppl 1995;520:256-57

7. Koizuka I, Seo R, Sano M, et al. High-resolution magnetic resonance imaging of the human temporal bone. ORL J Otorhinolaryngol Relat Spec 1991;53: 357-61

8. Naganawa S, Komada T, Fukatsu H, et al. Observation of contrast enhancement in the cochlear fluid space of healthy subjects using a 3D-FLAIR sequence at 3 Tesla. Eur Radiol 2006;16:733-37 